\title{
Médiations contrôlantes et structurantes: une base pour penser la formation
}

\section{Alexandre A.J. Buysse ${ }^{1}$}

En prenant appui sur une approche détaillée des régulations impliquées dans tout apprentissage, nous proposons une conceptualisation des médiations. Nous abordons le développement sous un angle vygotskien et abordons les mécanismes d'intériorisation et de poursuite de l'intériorisation qui le sous-tendent, ainsi que la notion de médiation. Nous distinguons les médiations contrôlantes et les médiations structurantes. Les médiations contrôlantes intériorisées influencent la manière dont l'apprenant contrôle les régulations et peuvent aboutir au développement d'un style d'apprentissage autorégulé. Les médiations structurantes intériorisées influencent la manière dont les apprenants comprennent et donnent un sens au savoir. En nous basant sur cette conceptualisation, nous esquissons des pistes pour penser la formation.

Cet article propose au lecteur une manière de penser les médiations dans le cadre des formations tant scolaires que professionnelles. Nous situons d'abord l'apprentissage et le développement dans un perspective vygotskienne. Considérant les systèmes psychologiques, nous tentons ensuite d'établir un lien avec les régulations et la réflexivité. Après avoir posé certaines caractéristiques des médiations, nous argumentons en faveur de l'établissement d'une distinction entre des médiations contrôlantes et des médiations structurantes. Finalement, sur cette base, nous lançons quelques suggestions pour la formation. Notre démarche est conceptuelle, nourrie toutefois par nos travaux antérieurs sur l'analyse des médiations dans le cadre scolaire et dans le cadre de la formation à l'enseignement.

\section{Apprentissage et développement}

Le savoir peut être considéré comme le résultat d'un processus historico-culturel, ayant amené une interprétation culturellement admise. Il se déploie dans la perspective particulière des évolutions historiques de chaque culture et dans le cadre plus restreint et transculturel de chaque discipline. Le savoir repose ainsi 
sur une épistémie particulière liée aux disciplines scientifiques à son origine. Il émerge aussi au sein de chaque profession dans le cadre de son adaptation historique à sa fonction sociale et à la logique de ses outils. À toutes ces déclinaisons culturellement établies de différents savoirs, s'ajoute un degré d'autonomie laissé au sujet dans le cadre de ses actions, de ses apprentissages. Cette autonomie inclut, à des degrés variables, la fixation des objectifs, les étapes devant faire l'objet d'une évaluation et les contrôles.

Si nous admettons ce point de vue, un apprentissage peut dès lors être vu comme un processus de prise de conscience subjective de la logique historico-culturelle dans laquelle les objets de savoir doivent se construire (Buysse \& Vanhulle, 2009; Vanhulle, 2008). Cette logique, propre à une discipline ou à une profession, se reflète entre autres dans les concepts structurant les savoirs, et dans la plus ou moins grande autonomie du sujet.

Sur cette base, nous pouvons distinguer:

1. l'apprentissage: intériorisation qui consiste dans le passage de l'interpsychique à l'intrapsychique, résultant en une appropriation des savoirs par le sujet;

2. le développement: la poursuite de l'intériorisation qui consiste en la restructuration des savoirs préexistants du sujet sur la base de ces nouveaux savoirs investis de sens. Il y a ainsi transformation du fonctionnement psychique du sujet (Vygotsky, 1997a).

Vue sous l'angle de l'analyse piagétienne (Inhelder \& de Caprona, 1992; Piaget, 1974, 1975), la compréhension d'un apprentissage - dans un premier temps synonyme de réussite - consiste à donner un sens. Donner un sens est ainsi l'aboutissement d'une abstraction réfléchissante résultant en un dépassement du système. Cette abstraction réfléchissante est l'œuvre du sujet et est liée à une autorégulation de sa part, même si à l'origine elle s'intègre dans un ensemble plus vaste de régulations largement hétérogènes.

Dans des études antérieures, portant sur le développement professionnel des enseignants, nous avons pu ainsi distinguer entre autres une acquisition, une appropriation et une subjectivation du savoir (Buysse \& Vanhulle, 2009).

L'acquisition consiste en l'assimilation d'un savoir sans que le système de signification interne de celui-ci ne soit compris par le sujet ou sans que le sujet ne lui ait donné un sens. Il y a appropriation lorsque le sujet comprend les liens de significations qui unissent les éléments du concept selon le cadre épistémique qui est propre à celui-ci. C'est le résultat de l'intériorisation des concepts par le sujet, donc de l'apprentissage. Le sujet comprend les significations internes d'un concept scientifique et peut les expliciter et en faire la synthèse.

La subjectivation a lieu lorsque le sujet donne un sens personnel au concept scientifique. Il est alors en mesure d'expliquer le concept en ses propres mots, de prendre une certaine distance avec le cadre historico-culturel et de donner au savoir un sens qui lui est propre tout en respectant sa logique constitutive. Lors du discours lié à une situation, le sujet explicite les concepts à sa manière et montre que ceux-ci ont un sens pour lui (Buysse \& Vanhulle, 2009). 
La subjectivation est ainsi vue comme liée à la création d'un sens propre au sujet suite à la poursuite de l'intériorisation au niveau intrapsychique sans que ce sens soit pour autant indépendant du sens culturellement établi (Gonzalez Rey, 2008). Il faudrait en effet que l'intériorisation se poursuive et rencontre l'autodéveloppement du sujet, son mécanisme naturel de développement, pour qu'il y ait développement (Brossard, 2004; Wertsch, 1985). Lors de cette phase, l'apprenant cherche à donner un sens personnel au savoir. Le résultat est une restructuration des savoirs préexistants de l'apprenant. Nous pouvons en effet supposer que, la plupart du temps, les fonctions sont les mêmes mais que les relations entre elles changent en développant de nouveaux systèmes psychologiques qui modifient les systèmes existants (Vygotsky, 1997c).

Ceci correspondrait à la rencontre entre les concepts quotidiens représentant le développement actuel de l'apprenant et les concepts scientifiques dont l'intériorisation établit en quelque sorte la limite supérieure de la zone proximale de développement (Vygostki, 1997a). La zone proximale de développement est liée à la fois à cette interaction interpsychique et au potentiel d'autodéveloppement du sujet. Le développement des concepts scientifiques avance plus vite que celui des concepts quotidiens. Le niveau de maîtrise des concepts quotidiens indique le niveau de développement actuel du sujet et le niveau de maîtrise des concepts scientifiques se situe au sommet de la zone de développement proximal.

Cette manière de concevoir le développement à travers la création d'un sens permet, à notre avis, de cerner la double appartenance des savoirs à la fois objectifs/sociaux et subjectifs/personnels.

Ce qui nous semble important dans cette notion de développement c'est que le sujet pourra par la suite atteindre d'autres apprentissages sans l'étayage d'un tiers. Il peut aller ailleurs que là où il aurait été auparavant. Il ne s'agit pas nécessairement d'une progression mais bien d'un changement qualitatif et visant l'autonomie. Nous voyons ici que le contrôle de ce processus doit être dévolu totalement à l'apprenant et qu'il devrait l'avoir intégré à son fonctionnement intrapsychique.

Soulignons que nous constatons que la plupart des concepts scientifiques sont d'abord l'objet d'une acquisition ou d'une appropriation. La subjectivation n'a pas nécessairement lieu, du moins dans un premier temps. Vygotsky (1978) soulignait déjà:

La transformation d'un processus interpersonnel en un processus intrapersonnel est le résultat d'une longue série d'événements développementaux. Le processus en cours de transformation continue pendant longtemps d'exister et de changer en tant que forme externe d'activité avant de se tourner entièrement vers l'intérieur. Pour de nombreuses fonctions le stade des signes externes se poursuit pour toujours, c'est-à-dire que c'est leur stade final de développement. D'autres fonctions se développent plus avant et deviennent progressivement des fonctions internes. Toutefois, elles ne prennent seulement le caractère d'un processus interne qu'à la suite d'un développement prolongé. Leur transfert vers l'intérieur est lié à des changements 
dans les lois qui gouvernent leur activité; elles sont incorporées à un nouveau système avec ces propres lois. (p. 57)

C'est souvent bien plus tard que l'internalisation porte ses fruits et qu'on peut constater une subjectivation. Nous avançons toutefois que ce processus naturel peut être influencé à travers des dispositifs conçus à cet effet (Buysse \& Vanhulle, 2009). Cette démarche est à notre avis essentielle car, d'un point de vue développemental, l'appropriation de concepts sans poursuite de l'intériorisation correspond à une sorte de schizophrénie dans laquelle le développement est limité à une partie du système de l'apprenant. Ceci ne veut pas dire que le développement devrait s'étendre à toutes les fonctions en même temps. Des différences existent quant au développement des différents systèmes (Wertsch \& Tulviste, 1992). L'ensemble du système psychologique n'est pas nécessairement modifié. Dans ce cas, certains liens s'effacent progressivement (Vygotsky, 1997c). Le sujet s'en remet à son habitus et se fie progressivement au fait que les routines fonctionnent sans qu'il n'ait plus besoin de s'interroger sur leurs significations. Le développement scolaire ou professionnel peut ainsi rester séparé du développement de la personne. Il ne prendrait toutefois sa pleine expansion qu'en modifiant et en étant influencé par l'ensemble du système psychologique de l'apprenant ${ }^{2}$.

Afin de comprendre les processus d'intériorisation, nous avons recours à la notion de régulation des systèmes. En effet, Vygotsky, tout en n'ayant pas eu le temps de continuer ses recherches, a ouvert la voie en s'intéressant à la régulation du comportement et en abordant le développement sous l'angle de systèmes psychologiques (Vygotski, 1997c).

Nous inspirant de la conceptualisation de la subjectivation de Gonzalez Rey (2008), nous pourrions voir le sujet comme étant en interaction permanente avec un système plus large qui l'englobe. Ce système correspondrait à la société, la culture et la situation dans lesquelles les actions du sujet sont inscrites.

Dans cette même perspective, nous pourrions considérer que le sujet luimême englobe:

1. plusieurs systèmes qui sont ouverts sur et en interaction avec ce système plus large que constitue l'environnement dans lequel le sujet agit. Ces systèmes comprendraient notamment un système d'apprentissage interrelié à un système d'émotions (Vygostky, 1998, 2005);

2. un système intrapsychique, sous-tendant ces systèmes propres au sujet et tissant des liens entre eux.

C'est entre le système d'apprentissage et le système qui l'englobe que se passe l'intériorisation depuis l'interpsychique. Le système psychologique du sujet, ou système développemental au sens large ${ }^{3}$, est, lui, en interaction constante avec son système d'apprentissage. La poursuite de l'intériorisation a lieu lors du passage de ce système d'apprentissage au système de développement du sujet. Cette intériorisation a donc lieu au niveau intrapsychique.

Or les systèmes s'ajustent afin de maintenir un équilibre et le processus de rétablissement de l'équilibre est à la base même du dynamisme de la pensée. La 
régulation d'un système entraîne le déséquilibre de l'autre et donc la nécessité de régulation de ce dernier.

\section{Régulations}

Les régulations sont en effet «les mécanismes qui assurent le guidage, le contrôle et l'ajustement des activités cognitives, affectives et sociales» (Allal, 2007, p. 9). Elles seraient donc ce qui assure l'adaptation du fonctionnement d'un système. Ainsi, les processus de régulation expliquent comment les perturbations sont traitées par le système pour revenir à un état d'équilibre (Piaget, 1975). Dans ce sens, nous pouvons voir toute tentative d'intériorisation comme une perturbation engendrant un déséquilibre plus ou moins important du système du sujet.

Les régulations entreprises par le sujet s'inscrivent néanmoins dans les régulations liées au système dans lequel il évolue, et sont plus particulièrement dépendantes de celles des formateurs qui interagissent avec lui. Même si une part au moins d'autorégulation, vue comme la part propre au sujet dans les régulations qu'il entreprend lors d'une intériorisation, semble nécessaire, elle ne se conçoit qu'en lien avec ces régulations externes au sujet (Allal \& Mottier Lopez, 2007; Allal \& Saada-Robert, 1992; Saada-Robert, 1994).

Les régulations jouent un rôle majeur dans l'acquisition des connaissances, mais elles sont aussi à la base d'une reconstruction lors des transferts, c'est-à-dire lors de l'utilisation de savoirs dans de nouveaux contextes grâce à la généralisation qui est liée à la poursuite de l'intériorisation à un niveau intrapsychique (Ghassemzadeh, 2005; Van Oers, 1998). Cette poursuite de l'intériorisation s'éloignant du système externe au sujet, semble reposer sur une part essentielle, voire exclusive, d'autorégulation. Mais nous reviendrons sur ce qui néanmoins continue de l'influencer.

Le processus de régulation peut se résumer à des opérations successives visant à fixer un objectif à atteindre, orienter l'action vers l'atteinte de l'objectif, contrôler la progression vers le but, confirmer ou modifier la procédure et/ou redéfinir l'objectif (Allal, 2007; Allal \& Saada-Robert, 1992; Buysse, 2007).

Nous pouvons distinguer les opérations de régulation selon:

1. les différentes opérations de contrôle et d'évaluation à l'œuvre dans les régulations. Elles sont plus ou moins autonomes et sont en général négociées avec le formateur ou déterminées culturellement;

2. les différentes opérations de sélection des procédures, de détermination des objectifs et d'anticipation du résultat visé. Un formateur peut apporter un éclairage sur les logiques et concepts menant à ces processus. Ceci aide le sujet à comprendre, à trouver des solutions face à des problèmes inédits et donc à intérioriser de nouveaux savoirs.

Les connaissances dont l'intériorisation est visée n'influencent pas la nature profonde de ces différentes opérations. Toutefois, le quoi et le comment de la régula- 
tion varient selon les disciplines scientifiques, selon le cadre conceptuel et la culture professionnelle. Sont ainsi culturellement déterminés: le problème à résoudre, les objectifs à atteindre, les résultats à obtenir, les contraintes à observer, les procédures à disposition, les habitus dans la manière d'en créer de nouvelles, les éléments à contrôler en priorité lors de la progression vers le but, les critères d'évaluation du résultat final, l'ajustement en priorité de la procédure ou de l'objectif. Les régulations se situent ainsi dans un environnement complexe et peuvent autant relever des régulations inhérentes à l'activité du sujet autorégulation - qu'être provoquées par un formateur, des pairs, la situation ou des outils (Allal \& Saada-Robert, 1992).

Ces différentes régulations n'auront toutefois un effet sur les apprentissages que si elles s'intègrent au processus d'autorégulation du sujet (Allal, 2007), et nous pouvons dès lors parler d'intériorisation. Il y aurait donc lors d'une intériorisation, un ensemble de régulations dont une part relève de l'autorégulation du sujet. Mais dans quelle mesure la régulation est-elle contrôlée par le sujet lors d'une intériorisation?

On peut distinguer, en fonction de la source du contrôle exercé sur le processus d'autorégulation, (Buysse, 2007, 2008; Iran-Nejad, 1990) des régulations:

1. directes, obéissant à un contrôle externe et à une hétérorégulation;

2. automatisées, dépendant de l'apprenant mais n'exigeant pas un contrôle attentionnel car ne présentant aucune difficulté;

3. actives, sous le contrôle attentionnel de l'apprenant, car présentant en général des difficultés;

4. dynamiques, dépendant de l'apprenant mais hors de son contrôle attentionnel, lors d'un processus de régulation souvent différé qui reconceptualise, innove, tisse des liens entre ce qui est appris et les connaissances de l'apprenant. Ces différents contrôles se complètent et s'exercent sur des opérations différentes du processus de régulation. Le contrôle de la régulation semble différer également selon qu'il s'agit d'une régulation liée à l'intériorisation du savoir ou à la poursuite de l'intériorisation au niveau intrapsychique.

En effet, le sujet fait preuve de régulation active dans la phase d'intériorisation d'un savoir ou lorsqu'il recherche une solution. Les opérations de régulation impliquées étant multiples, une partie des tâches non-problématiques sont déléguées à des sous-processus de régulations automatisées, donc implicites. Le sujet peut ainsi dédier son attention au processus problématique. Cette régulation active, objet de la plupart des études métacognitives, opère à travers des régulations volontaires le plus souvent explicites et instrumentées (Allal \& Saada-Robert, 1992).

Quand le sujet ne parvient pas à trouver immédiatement une solution, il semble déléguer une partie des processus de régulation vers une régulation dynamique opérant en dehors de son contrôle attentionnel (Iran-Nejad \& Chissom, 1992). Des liens seraient ainsi établis entre des savoirs existants et les nouvelles données, sans que le sujet puisse expliciter l'ensemble de ce processus. Ces régu- 
lations semblent également permettre l'intégration progressive des nouvelles découvertes résultant des régulations actives de l'apprenant. La poursuite de l'intériorisation au niveau intrapsychique semble ainsi principalement liée à des régulations dynamiques.

Nous considérons qu'une part au moins de régulation active intervient dans toute action et dans tout apprentissage, et qu'une part importante de régulation dynamique a lieu lors de la poursuite de l'intériorisation.

La régulation active, par la conscience du problème, agit ainsi comme un organisateur des processus non conscients à l'œuvre dans la régulation dynamique. Ces processus non conscients sont conditionnés par les «actes préalables de connaissance rationnelle, de compréhension, de reconnaissance, d'association» (Vygotski, 2005, p. 357) et dépendent de la direction que l'on donne aux processus conscients.

L'intériorisation semble donc dépendre de régulations contrôlées de manière plus ou moins automatisée ou intentionnelle. Ces régulations sont la plupart du temps l'objet d'une réflexivité de la part du sujet. Nous pouvons nous interroger sur le rôle joué dans le processus de régulation par la réflexivité afin de mieux tenter de cerner l'intériorisation des savoirs.

La réflexivité est un retour sur la pensée elle-même, mais peut aussi impliquer des savoirs que le sujet mobilise pour pouvoir réguler. La fonction de la réflexivité n'est pas identique selon les étapes du processus de régulation. La réflexivité assume en effet deux fonctions:

1. la fonction de réflexivité contrôlante au sens d'abstraction réfléchissante, correspondant à la prise de conscience du processus de régulation;

2. la fonction de réflexivité structurante au sens d'abstraction réfléchie (Piaget, 1974) pouvant inclure la:

- prise en compte de différents savoirs et informations afin de réorienter le processus de régulation;

- consolidation de la régulation aboutie et ainsi de l'appropriation de nouveaux savoirs;

- restructuration des savoirs préexistants chez le sujet.

La réflexivité contrôlante, à travers la prise de conscience des régulations, permet au sujet d'évoluer vers un apprentissage autorégulé. En effet, celui-ci se dessine dans un contexte donné, dans l'interaction entre les régulations proposées par le formateur ou les autres apprenants et l'autorégulation du sujet (Buysse, 2007).

Pareillement, à travers la réflexivité structurante, les régulations sont intimement liées aux savoirs préexistants et aux savoirs et concepts proposés par le formateur ou les pairs dans un contexte donné. De cette interaction découle la capacité cognitive du sujet à atteindre des régulations conformes et efficientes par rapport au contexte disciplinaire ou professionnel et à intérioriser les savoirs. On peut également voir cette réflexivité structurante comme étant ce qui permet le développement du sujet. Il importe donc de considérer comment les médiations agissent sur les régulations et la réflexivité. 


\section{Médiations}

Si nous admettons que le sujet intériorise principalement des savoirs culturellement établis, nous pouvons considérer que les savoirs ne font pas l'objet d'une intériorisation directe. Toute opération d'intériorisation ${ }^{4}$ semble en effet impliquer un troisième élément, médiateur. Même pour la mémorisation, l'intériorisation ne peut être qu'indirecte, médiate (Vygotsky, 1997e).

Les médiations sont des outils psychologiques qui s'inscrivent dans une culture donnée. Elles existent d'abord dans l'interaction avant d'être intériorisées. Lors de l'intériorisation, elles deviennent progressivement un moyen utilisé par le sujet pour piloter ses processus psychiques. Cette intériorisation se poursuit et la nécessité d'un moyen-stimulus externe cesse d'exister. La poursuite de l'intériorisation ne peut toutefois avoir lieu que si cette médiation conserve une partie de ses propriétés. Cette médiation dérivée est en quelque sorte devenue propre à l'apprenant et s'est intégrée au savoir intériorisé.

\section{Le caractère dialectique}

Une des caractéristiques principales des médiations sont les propriétés dialectiques nécessaires à l'appropriation. En effet, un système conceptuel ne peut faire l'objet d'une intériorisation en bloc mais les tensions entre ses composantes doivent être rendues apparentes dans la richesse de leurs rapports dialectiques. La conception dialectique des processus du développement semble fondamentale (Brossard, 2004; Wertsch, 1985; Wertsch \& Tulviste, 1992). Cette conception renforce la nécessité d'une distinction entre un processus premier d'intériorisation et un processus subséquent de poursuite de l'intériorisation au niveau entièrement intrapsychique.

Le moteur de toute dialectique repose sur la recherche de sens par le sujet: la signification, «c'est la structure interne de l'opération sémiotique. C'est ce qui existe entre la pensée et le mot» (Vygotsky, 1997d, p. 133). Il y a tension entre une signification attribuée au signe, quels que soient sa nature et son lien au réel, et le sens qu'il prend ou tend à prendre dans l'interprétation contextuelle immédiate (Valsiner, 2002). Ce contexte est soit l'environnement dans le cas d'un savoir à construire, soit le système conceptuel lui-même dans le cadre des savoirs établis. Le sens que prend le signe est donc par essence dialectique. S'il n'en était pas ainsi, la dynamique nécessaire à l'intériorisation n'existerait pas ni celle nécessaire à la poursuite de l'intériorisation.

Il en découle que la médiation doit avant tout permettre l'intériorisation de ces rapports (Karpov \& Haywood, 1998). Ces médiations ne facilitent pas seulement les processus mais «en étant inclus dans le processus comportemental, les outils psychologiques altèrent l'ensemble du flux et de la structure des fonctions psychologiques» (Wertsch \& Tulviste, 1992, p. 551). Elles sont le produit d'une évolution socioculturelle et font l'objet d'une appropriation par le sujet dans son fonctionnement psychologique. Ceci conduit Vygotski à considérer la culture 
comme un système de signes qui crée des formes particulières de comportement, modifie le fonctionnement psychologique et construit de nouvelles histoires dans le système du comportement humain en développement (Wertsch \& Tulviste, 1992).

La médiation ne consiste ainsi pas simplement en une association avec un savoir mais modifie la manière dont ce savoir est intériorisé. Il ne s'agit pas d'associer un élément avec un autre mais de combiner les éléments entre eux, la médiation influençant durablement, restructurant en quelque sorte, le savoir qui est objet de la médiation. Toute appropriation d'un savoir entraîne ainsi immanquablement sa transformation par le sujet (Vanhulle, 2009). Les outils psychologiques influencent ainsi le processus psychologique et en changent la structure.

Nous en concluons qu'il ne peut y avoir d'intériorisation durable sans que:

1. les savoirs n'aient été influencés par la médiation;

2. les médiations soient intériorisées simultanément et liées aux savoirs;

Et, dans ce cadre:

3. les médiations relèvent d'environnements culturels;

4. les médiations sont influencées par les contextes d'intériorisations et d'utilisation des médiations.

Les médiations ne sauraient se réduire à la matérialité d'un support, artefact (livre, données, image) ou même à l'interaction (formateur), que nous appellerons vecteurs de la médiation, car le sujet intériorise la médiation et pas le médiateur (Kozulin \& Presseisen, 1995). Ce vecteur est nécessaire au transfert mais la médiation dépend plutôt de ses caractéristiques propres. C'est ainsi qu'ont été abondamment étudiés d'une part le contexte dans lequel les médiations se déploient et, d'autre part, la médiation des outils et des signes (Bernié, 2001; Bronckart, 1996; Daniels, 2008; Moro, Schneuwly \& Brossard, 1997).

Si nous admettons, tel que nous l'exposions plus haut, que l'intériorisation d'un savoir provoque des déséquilibres dans le système du sujet, il peut être intéressant d'examiner les médiations selon leur influence sur les régulations. De plus, si, comme présenté au début de cet article, nous considérons que l'apprentissage et le développement sont liés, nous devrions prendre en compte le rôle développemental de la médiation.

\section{Deux types de médiations}

Nous proposons de distinguer, en fonction de leur influence sur les régulations et de leur portée développementale, des médiations contrôlantes et des médiations structurantes 5 .

\section{Médiations contrôlantes}

Lors de l'enseignement/apprentissage, le sujet bénéficie d'un certain nombre 
d'hétérorégulations portant sur le processus de régulation durant l'intériorisation. Il s'agit de contrôles externes d'étapes de la régulation exercés en général par un formateur, mais aussi par différents facteurs contextuels hors du contrôle de l'apprenant. La médiation porte ainsi une composante que nous qualifierons de médiation contrôlante dans la mesure où elle exerce une hétérorégulation sur les régulations de l'apprenant. Cette distinction nous permet de cerner la médiation qui est intériorisée en tant que contrôle intrapsychique des régulations. Elle peut toucher à la fois à la fixation de l'objectif, au contrôle du processus de régulation ou à l'évaluation.

Ces médiations que nous qualifierons de contrôlantes sont intériorisées par l'apprenant et font dès lors partie de son fonctionnement, indissociablement liées aux savoirs intériorisés.

L'intériorisation de la médiation contrôlante est liée à la communication interpersonnelle qui est intériorisée progressivement en tant qu'outil sémiotique d'autorégulation dans le langage intérieur (Karpov \& Haywood, 1998). Mais la complexité de la communication dans l'enseignement/apprentissage fait que cette intériorisation ne résulte pas nécessairement en un autocontrôle, les diverses médiations favorisant plus ou moins fortement les autorégulations du sujet.

La poursuite de l'intériorisation de la médiation contrôlante au niveau intrapsychique engendrerait un style d'apprentissage plus ou moins autonome ou dépendant. Ceci se produirait selon les rapports entre la médiation contrôlante et l'autodéveloppement de l'apprenant. À cet égard, Vermunt et Verloop (1999) démontrent un jeu de congruence et de friction selon la mesure dans laquelle le partage du contrôle entre le formateur et l'apprenant est adapté au style de ce dernier. De la qualité de cette rencontre résulterait le développement d'un style d'apprentissage, allant d'une dépendance aux contrôles externes à un style d'apprentissage autorégulé.

Dans le discours des étudiants en formation à l'enseignement, nous constatons (Buysse \& Vanhulle, 2009) une médiation contrôlante: par les formateurs, par les prescriptions, par la théorie, par les objectifs, par le contexte, par l'anticipation. La référence à un contrôle par la situation ou par anticipation du sujet lui-même, indique une médiation contrôlante subjectivée par le sujet. Nous avons aussi pu observer des médiations contrôlantes de la part du formateur dans le cas de l'enseignement/apprentissage auprès d'élèves du cycle primaire (Buysse, 2007).

\section{Médiations structurantes}

Parallèlement à cette médiation contrôlante, ou indépendamment d'elle, une médiation structurante est intériorisée et modifie les processus de régulation eux-mêmes. Cette médiation peut être vue comme les outils cognitifs nécessaires à l'intériorisation et à la poursuite de l'intériorisation.

Si nous considérons que la médiation permet l'intériorisation, donc favorise le processus de régulation, nous pouvons émettre l'hypothèse qu'elle influence: 
les différentes opérations de sélection des procédures, la détermination des objectifs et l'anticipation du résultat visé ainsi que principalement la fonction de réflexivité structurante liée à la régulation. En un mot, elle permettrait au sujet de comprendre le savoir à intérioriser et ensuite de le subjectiver. La médiation comporterait ainsi une composante que nous qualifierons de médiation structurante.

Les savoirs scientifiques véhiculés au sein d'une discipline ou d'une culture professionnelle reposent sur un système particulier, une épistémie, qui assure leur cohérence (Buysse, sous presse). La culture conditionne aussi la manière dont les régulations s'effectuent afin de trouver des actions appropriées à de nouvelles situations. Le processus de résolution de problèmes apparent dans le travail des professionnels et des formateurs, l'agencement particulier des systèmes conceptuels, cette weltanschauung, sont autant de médiations structurantes.

La médiation structurante qui est présente dans le discours particulier de présentation d'un savoir est intériorisée en tant que manière de réguler. Elle influence durablement les savoirs lors de leur intériorisation et permet de les comprendre.

La poursuite de l'intériorisation de la médiation structurante doit permettre de remettre en question le sens donné aux savoirs. Elle doit permettre d'aménager ce sens pour tenir compte du contexte intrapsychique de l'apprenant, de ses valeurs, de ses affects, de ses savoirs préexistants, sans perdre totalement ses caractéristiques. Les concepts scientifiques, en tant que médiation structurante, jouent ici un rôle déterminant car ils représentent la généralisation de l'expérience de l'humanité et une fois intériorisés deviennent les outils psychologiques nécessaires à la résolution de problèmes (Karpov, 2003; Karpov \& Haywood, 1998; Kozulin \& Presseisen, 1995).

Les propriétés déterminantes des concepts scientifiques sont en effet leur caractère explicite, un système d'organisation systémique, logique et hiérarchique, ainsi que leur utilisation délibérée. Idéalement, les concepts scientifiques reflètent ce qui est vu comme l'essence d'un certain aspect de la réalité et se distinguent par les liens entre les éléments qui les composent. Ils forment un système socio-culturellement validé (Van der Veer, 1998). Ceci les distingue des concepts quotidiens ayant leur origine dans l'interprétation que fait l'individu des événements, même si cette interprétation est elle-même la conséquence des différents savoirs et modes de pensées ayant co-construit le sujet.

Nous pouvons considérer qu'apprendre dans une perspective développementale demande une intériorisation de savoirs grâce à la médiation structurante de leur épistémie conceptuelle. Notons que la médiation structurante semble pouvoir être dégagée d'un savoir par un sujet ayant développé un style d'apprentissage autorégulé. Elle n'est donc pas toujours portée par le formateur directement, contrairement à la médiation contrôlante qui ressort le plus souvent d'une interaction. S'ajoute à cela que la tension dialectique de la médiation tant entre l'individu et la médiation, qu'au sein de l'individu lui-même lors de l'utilisation de 
l'outil psychologique dans une situation particulière, appellent la créativité personnelle du sujet. Vu que les actions du sujet se situent toujours dans des contextes changeants, cela implique une utilisation créative de ces médiations structurantes qui restent toutefois conditionnées de manière fondamentale par leur origine culturelle ou épistémique (Wertsch \& Tulviste, 1992).

Dans le cadre d'un enseignement scolaire, nous pouvons envisager la médiation structurante comme idéalement constituée de l'explication conceptuelle sous-tendant le savoir à intérioriser, de la démonstration du sens que peut prendre ce savoir (Inagaki, Miyake \& Cole, 2007). À défaut, elle consisterait en la mise à disposition d'outils permettant de la révéler au sujet indirectement. Au niveau scolaire, l'effet des médiations structurantes s'observe notamment lorsque, suite à l'explication des concepts à la base d'un savoir, l'élève parvient à réinvestir ce savoir dans des situations non identiques (Karpov, 2003).

Dans le cadre de la formation professionnelle, il s'agirait de faire apparaître la logique professionnelle permettant l'élaboration des savoirs professionnels. L'intériorisation de la médiation structurante apparaît ainsi, par exemple, dans le discours des étudiants en formation à l'enseignement, en tant que référence à des cadres théoriques appropriés, à la fixation d'objectifs de régulation conformes à la profession (Buysse \& Vanhulle, 2009).

\section{Quelques pistes pour la formation}

Nous considérons donc qu'il peut être utile de favoriser au niveau de la formation:

1. les médiations contrôlantes qui permettraient de donner une part de contrôle des apprentissages au sujet et de favoriser le développement d'un style d'apprentissage autorégulé;

2. les médiations structurantes qui permettraient de donner un sens aux savoirs et de favoriser le développement du sujet.

C'est afin de favoriser l'intériorisation des médiations contrôlantes, dans le sens d'un contrôle par le sujet de l'ensemble du processus, que certains dispositifs de formation portent sur un travail de questionnement plutôt que sur des injonctions. Il est vrai que, quoi qu'il arrive, la médiation contrôlante existe à travers la communication entre l'enseignant et le sujet. Le sujet prend par exemple ses repères dans les attentes du formateur, et détermine ce qu'il lui semble pouvoir contrôler de manière autonome dans toute tâche. Il intériorise ainsi un degré d'autonomie.

Du point de vue des médiations structurantes, une plus grande prise de conscience est nécessaire. Le sujet peut éventuellement les dégager lui-même de dispositifs didactiques, mais ils doivent être pensés par le formateur à cette fin. Il conviendrait de penser une formation forte en concepts scientifiques et prévoyant l'explicitation de l'épistémie de ceux-ci. À travers cela, il faut permettre 
aux étudiants de répondre à des questions portant sur le "pourquoi», de défendre leur manière personnelle de résoudre des problèmes et les résultats obtenus. Une formation donnant une importance accrue à la médiation de l'épistémie des concepts scientifiques amènerait les étudiants à tenter de trouver dès le début les caractéristiques essentielles du problème, sans s'arrêter aux caractéristiques superficielles de la situation (Karpov \& Haywood, 1998). Dans ce cadre, il convient aussi d'inclure dans les dispositifs des conditions favorables à la créativité du sujet et donc au développement des régulations dynamiques nécessaires à la poursuite de l'intériorisation. Celles-ci comprennent un climat détendu mais surtout des interactions particulières entre formateur et sujet basées sur des questionnements ouverts et la recherche conjointe de réponses.

Les concepts scientifiques sont bien loin des concepts quotidiens, et pourtant les savoirs doivent devenir personnels par leur mise en pratique. Ceci dépend toutefois de la mesure dans laquelle on peut expliciter les savoirs (Van der Veer, 1998). Il nous semble important de redonner une pleine valeur à l'explication des concepts sous-tendant les savoirs enseignés, et partant de valoriser les concepts scientifiques en tant que médiation structurante.

En conclusion, nous pensons que l'analyse des dispositifs sous l'angle des médiations contrôlantes et structurantes peut amener de nouveaux développements. Ceux-ci devraient reposer sur les acquis du socioconstructivisme qui a mis l'accent en occident sur la médiation contrôlante et sur les développements plus historico-culturels des théories vygotskiennes qui ont œuvré à la création de dispositifs orientés vers les médiations structurantes. Une formation des enseignants exploitant ces deux médiations pourrait être une première étape dans ce sens.

\section{Notes}

1 Section des sciences de l'éducation, groupe de Recherche Théorie, Action, Langage et Savoirs (TALES) dirigé par Prof. S. Vanhulle.

2 Voir les besoins d'actualisation, de congruence du sujet, et l'apprentissage transformationnel (Mezirow, 2001).

3 Comprenant également les émotions et autres aspects subjectifs; voir Vygotsky(1997b).

4 Ici nous excluons volontairement la mémorisation sensori-motrice et la mémorisation à court terme pour lesquels une intériorisation «directe» est envisageable quoique là-aussi peu efficiente.

5 Nous ne reprenons pas la distinction de Karpov et Haywood (1998) qui parlent respectivement de médiations métacognitives et cognitives. Si elle rend bien compte de deux ordres de médiations présentant des similarités avec notre proposition en ce qui concerne le passage de l'interpsychique à l'intrapsychique, elle ne s'étend pas à l'aspect développemental. D'autre part, nous pensons que la qualification de métacognitive suggère l'existence d'un processus explicitable en opposition avec un processus cognitif qui le serait moins, ou indique à tout le moins une différence de niveau. Ceci nous semble en contradiction avec l'aspect profondément implicite de la poursuite de l'intériorisation de tout type de médiation. 


\section{Références bibliographiques}

Allal, L. (2007). Régulations des apprentissages: orientations conceptuelles pour la recherche et la pratique en éducation. In L. Allal \& L. Mottier Lopez (Éd.), Régulations des apprentissages en situation scolaire et formation (pp. 7-23). Bruxelles: De Boeck.

Allal, L. \& Mottier Lopez, L. (Éd.). (2007). Régulations des apprentissages en situation scolaire et formation. Bruxelles: De Boeck.

Allal, L. \& Saada-Robert, M. (1992). La métacognition: cadre conceptuel pour l'étude des régulations en situation scolaire. Archives de Psychologie 60, 265-296.

Bernié, J.-P. (Éd.). (2001). Apprentissage, développement et significations. Bordeaux: Presses universitaires.

Bronckart, J.-P. (1996). Activité langagière, textes et discours. Pour un interactionnisme socio-discursif. Neuchâtel: Delachaux et Niestlé.

Brossard, M. (2004). Vygotsky: Lectures et perspectives de recherches en éducation. Villeneuve d'Ascq: Presses Universitaires du Septentrion.

Buysse, A. (2007). Le théâtre: au-delà du jeu. Une exploration de la médiation de l'apprentissage autorégulé par la forme théatrale (Cahiers de la section des sciences de l'éducation No 114). Université de Genève.

Buysse, A. (2008). La médiation exercée par le formateur de théâtre sur la régulation dynamique des apprenants. In L. Mottier Lopez, Y.-E. Dizerens, G. Marcoux \& A. Perréard Vité (Éd.), Entre la régulation des apprentissages et le pilotage des systèmes: évaluations en tension. Actes du 20e colloque ADMEE-Europe, Université de Genève consulté le 20 janvier 2009 dans https://plone.unige.ch/sites/admee08/communications-individuelles/m-a $9 / \mathrm{m}$ a9-1/

Buysse, A. (sous presse). Une modélisation des régulations et de la médiation dans la construction des savoirs professionnels des enseignants. In P. Maubant \& S. Martineau (Éd.), Fondements des pratiques professionnelles des enseignants: pour une lecture compréhensive des fondements des savoirs professionnels des enseignants. Ottawa: Presses Universitaires d'Ottawa.

Buysse, A. \& Vanhulle, S. (2009). Écriture réflexive et développement professionnel: quels indicateurs? Questions Vives, 11 (5), 225-245.

Daniels, H. (2008). Reflections on points of departure in the development of sociocultural and activity theory. In B. Van Oers, W. Wardekker, E. Elbers \& R. Van der Veer (Éd.), The transformation of learning: advances in cultural-historical activity theory (pp. 58-75). New York: Cambridge University Press.

Ghassemzadeh, H. (2005). Vygotsky's mediational psychology: A new conceptualization of culture, signification and metaphor. Language Sciences, 27, 281-300.

Gonzalez Rey, F. L. (2008). Subject, subjectivity, and development in cultural-historical psychology. In B. Van Oers, W. Wardekker, E. Elbers \& R. Van der Veer (Éd.), The transformation of learning: advances in cultural-historical activity theory (pp. 137-154). New York: Cambridge University Press.

Inagaki, K., Miyake, N. \& Cole, M. (Éd.). (2007). Understanding human development as the emergent product of dynamic intra-mental/inter-mental interaction: a tribute to Giyoo Hatano.Human Development, 50, 1.

Inhelder, B. \& de Caprona, D. (1992). Vers le constructivisme psychologique: structures? procédures? Les deux indissociables. In Le cheminement des découvertes de l'enfant (pp. 19-50). Neuchâtel: Delachaux et Niestlé.

Iran-Nejad, A. (1990). Active and dynamic self-regulation of learning processes. Review of Educational Research, 60 (4), 573-602.

Iran-Nejad, A. \& Chissom, B. S. (1992). Contributions of active and dynamic selfregulation to learning. Innovative Higher Education 17, 125-136.

Karpov, Y. V. (2003). Internalization of children's problem solving and individual differences in learning. Cognitive Development 18, 377-398. 
Karpov, Y. V. \& Haywood, H. C. (1998). Two ways to elaborate Vygotsky's concept of mediation. American Psychologist 53 (1), 27-36.

Kozulin, A. \& Presseisen, B. Z. (1995). Mediated learning experience and psychological tools: Vygotsky's and Feuerstein's perspectives in a study of student learning. Educational Psychologist 30 (2), 67-75.

Mezirow, J. (2001). Penser son expérience: développer l'autoformation. Lyon: Chronique sociale.

Moro, C., Schneuwly, B. \& Brossard, M. (Éd.). (1997). Outils et signes: perspectives actuelles de la théorie de Vygotsky. Bern: Peter Lang.

Piaget, J. (1974). Réussir et comprendre. Paris: PUF.

Piaget, J. (1975). L'équilibration des structures cognitives: problème central du développement. Paris: PUF.

Saada-Robert, M. (1994). Microgenesis and situated cognitive representations. In N. Mercier \& C. Coll (Éd.), Explanations in socio-cultural studies 5 (pp. 55-64). Madrid: Infancia y aprendizaje.

Valsiner, J. (2002). Forms of dialogical relations and semiotic autoregulation within the self. Theory \& Psychology, 12 (2), 251-265.

Van der Veer, R. (1998). From concept attainment to knowledge formation. Mind, Culture, and Activity, 5 (2), 89-94.

Van Oer, B. (1998). The fallacy of decontextualization. Mind, Culture, and Activity, 5 (2), 135-142.

Vanhulle, S. (2008). Au cœur de la didactique professionnelle, la subjectivation des savoirs. In P. Pastré \& Y. Lenoir (Éd.), Didactique professionnelle, didactique des savoirs professionnels (pp. 227-254). Toulouse: Octarès.

Vanhulle, S. (2009). Des savoirs en jeu au savoir en je: Cheminements réflexifs et subjectivation des savoirs chez de jeunes enseignants en formation. Bern: Peter Lang.

Vermunt, J. D. \& Verloop, N. (1999). Congruence and friction between learning and teaching. Learning and Instruction, 9 (3), 257-280.

Vygotski, L. (1978). Mind in society: the development of higher psychological processes. London: Harvard University Press.

Vygostki, L. (1997a). Pensée et langage. Paris: La dispute.

Vygotski, L. (1997b). The collected works of L. S. Vygotsky (vol. 3): Problems of the theory and history of psychology (R. Van der Veer, trad.). New York: Plenum.

Vygotski, L. (1997c). On psychological systems (R. Van der Veer, trad.). In R. W. Rieber \& J. Wollock (Éd.), The collected works of L. S. Vygotsky (vol. 3): Problems of the theory and history of psychology (pp. 91-108). New York: Plenum.

Vygotski, L. (1997d). The problem of consciousness (R. Van der Veer, trad.). In R. W. Rieber \& J. Wollock (Éd.), The collected works of L.S. Vygotsky (vol. 3): Problems of the theory and history of psychology (pp. 129-138). New York: Plenum.

Vygotski, L. (1997e). The collected works of L. S. Vygotsky (vol. 4): The history of the development of higher mental functions (M. J. Hall, trad.). New York: Plenum.

Vygostki, L. (1998). Théorie des émotions. Paris: L’Harmattan.

Vygotski, L. (2005). Psychologie de l'art. Paris: La Dispute.

Wertsch, J. V. (1985). Vygotsky and the social formation of mind. Cambridge: Harvard University Press.

Wertsch, J. V. \& Tulviste, P. (1992). L. S. Vygotsky and contemporary developmental psychology. Developmental Psychology, 28 (4), 548-557.

Mots clés: Médiation, autorégulation, concept, développement, formation 


\section{Kontrollierende und strukturierende Vermittlungen: eine Basis für die Gestaltung der Ausbildung}

\section{Zusammenfassung}

Ausgehend von einer eingehenden Betrachtung der in jedem Lernprozess involvierten Regulierungen diskutiert der Beitrag eine Konzeptualisierung der Vermittlungen. Die Entwicklung wird in diesem Artikel in einer Vygotskij'schen Perspektive betrachtet, wobei insbesondere die Mechanismen der Verinnerlichung und der weiteren Vertiefung der ihr zugrunde liegenden Verinnerlichung sowie der Begriff der Vermittlung thematisiert werden. Unterschieden wird zwischen kontrollierenden Vermittlungen und strukturierenden Vermittlungen. Die verinnerlichten kontrollierenden Vermittlungen beeinflussen die Art und Weise, wie Lernende die Regulierungen überprüfen. Diese können zur Entwicklung eines selbstregulierten Lernstils führen. Die verinnerlichten strukturierenden Vermittlungen wirken auf die Art und Weise ein, wie Lernende verstehen und dem Wissen einen Sinn geben. Auf der Basis dieser Konzeptualisierung werden Anregungen zur Gestaltung der Ausbildung von Lehrerinnen und Lehrern dargelegt.

Schlagworte: Vermittlung, Selbst-Regulation, Konzept, Entwicklung, Ausbildung von Lehrpersonen

\section{Mediazioni, regolazioni e sviluppo: una base per pensare la formazione degli insegnanti.}

\section{Riassunto}

Basandosi su un approccio dettagliato delle regolazioni implicate in ogni apprendimento, l'articolo propone una concettualizzazione delle mediazioni. Si affrontano lo sviluppo da un punto di vista vygotskiano, i meccanismi d'interiorizzazione o di ricerca dell'interiorizzazione che lo caratterizzano e al tempo stesso la nozione di mediazione. La distinzione tra mediazioni controllanti e strutturanti è centrale: le prime, interiorizzate, influenzano la maniera in cui lo studente controlla le regolazioni e possono condurre a sviluppare uno stile d'apprendimento autoregolato. Le strutturanti seconde, interiorizzate, influenzano invece la maniera in cui gli studenti comprendono e danno un senso al sapere. Basandoci su questa concettualizzazione, abbozzeremo alcune piste per pensare la formazione

Parole chiave: Mediazione, autoregolazione, concetto sviluppo, formazione 


\title{
Controlling and structuring mediations: a way of thinking about teacher education
}

\begin{abstract}
Based on a detailed approach to the system regulations of teaching, we propose an ideation/conceptualization of mediations. Approaching this development from a Vygotskian perspective, we detail the pursuit of internalization, which enables the mechanisms of internalization, as well as the notion of mediation. We distinguish between controlling and structuring mediations. Once internalized, controlling mediations influence how learners control regulations which can develop into a self-regulated learning style. Internalized structuring mediations influence how learners make sense of knowledge. Based on this conceptualization, we outline new ways of thinking about education.
\end{abstract}

Key words: Mediation, self-regulation, concept, development, education 